\title{
STAT3-enhancing germline mutations contribute to tumor-extrinsic immune evasion
}

\author{
Daniel Kogan, ${ }^{1,2}$ Alexander Grabner, ${ }^{3}$ Christopher Yanucil, ${ }^{4}$ Christian Faul, ${ }^{4}$ and Vijay Kumar Ulaganathan ${ }^{5}$ \\ ${ }^{1}$ Technische Universität München, Munich, Germany. '2udwig-Maximilians-Universität, Munich, Germany. ${ }^{3}$ Division of Nephrology, Department of Medicine, Duke University Medical Center, Duke University, \\ Durham, North Carolina, USA. ${ }^{4}$ Division of Nephrology, Department of Medicine, The University of Alabama at Birmingham, Birmingham, Alabama, USA. ${ }^{5}$ Max Planck Institute of Biochemistry, \\ Department of Molecular Biology, Martinsried, Germany.
}

\begin{abstract}
Immune evasion and the suppression of antitumor responses during cancer progression are considered hallmarks of cancer and are typically attributed to tumor-derived factors. Although the molecular basis for the crosstalk between tumor and immune cells is an area of active investigation, whether host-specific germline variants can dictate immunosuppressive mechanisms has remained a challenge to address. A commonly occurring germline mutation (c.1162G >A/rs351855 G/A) in the FGFR4 (CD334) gene enhances signal transducer and activator of transcription 3 (STAT3) signaling and is associated with poor prognosis and accelerated progression of multiple cancer types. Here, using rs351855 SNP-knockin transgenic mice and Fgfr4-knockout mice, we reveal the genotype-specific gain of immunological function of suppressing the CD8/ $\mathrm{CD}^{+}{ }^{+} \mathrm{FOXP3}{ }^{+} \mathrm{CD} 25^{+}$regulatory $\mathrm{T}$ cell ratio in vivo. Furthermore, using knockin transgenic mouse models for lung and breast cancers, we establish the host-specific, tumor-extrinsic functions of STAT3-enhancing germline variants in impeding the tumor infiltration of CD8 T cells. Thus, STAT3-enhancing germline receptor variants contribute to immune evasion through their pleiotropic functions in immune cells.
\end{abstract}

\section{Introduction}

Immune evasion is considered the hallmark of cancers (1). Strategies that restore the capacity of the immune system to recognize and eliminate malignant cells have produced clinical benefits. However, due to a dearth of predictive biomarkers for patient stratification, only one-third of all patients are responsive to treatment (2). Immune evasion by tumor tissues has been the major bottleneck in the development of therapeutically effective anticancer strategies. The prominent mechanisms by which tumors evade immune attack include the evolution of tumor cell variants that are resistant to immune effectors and the progressive formation of an immune suppressive microenvironment within the tumor that impedes the infiltration of antitumor effector cells. Great emphasis has been placed on understanding the function of tumor-intrinsic somatic heterogeneity or the tumor-induced microenvironment in evading immune surveillance, but the role played by tumor-extrinsic, host-specific genetic heterogeneity in modulating the antitumor immune response is poorly understood and remains challenging to address.

An attribute common to all cancers is the presence of numerous cell types, including bone marrow-derived inflammatory cells, lymphocytes, fibroblastic cells, and the extracellular matrix composed of collagen and proteoglycans. Importantly, of diverse assemblages of tumor cell infiltrates, cytotoxic and regulatory $\mathrm{T}$

Conflict of interest: CF has served as a consultant or received honoraria from Ultragenyx and received research support from U3 Pharma $\mathrm{GmbH}$ and Roche.

Submitted: August 10, 2017; Accepted: February 8, 2018

Reference information: J Clin Invest. 2018;128(5):1867-1872.

https://doi.org/10.1172/JCI96708. lymphocytes within the tumors are often the crucial factors that determine the outcome of anticancer therapy $(3,4)$. For example, the increased $\mathrm{T}$ cell numbers, particularly an increased ratio of $\mathrm{CD} 8 / \mathrm{FOXP}^{+}$regulatory $\mathrm{T}$ cells (Tregs) within the tumor microenvironment (TME), predict a favorable therapeutic response, whereas severe lymphopenia negatively impacts the chemo- and immunotherapy response (5). It is well recognized that while the host immune system can recognize and reject cancerous cells, it can also mold the somatic heterogeneity of tumors by assisting in the generation of immune-resistant tumor variants (6). Various mechanisms, such as immunoediting, exist whereby primary tumor rejection is rendered compromised and ineffective by the inhibition of cytotoxic CD8 $\mathrm{T}$ cell infiltration or viability in the TME. An imminent question thus arises: can individual-specific heritable genetic variants regulate immune homeostasis such that immune surveillance is impaired in a host-dependent manner irrespective of the nature of oncogenic onslaught? Here, we attempted to address this question by dissecting the tumorextrinsic immunological function of signal transducer and activator of transcription 3-enhancing (STAT3-enhancing) germline receptor variants in shaping the TME. Many of the genes that have been studied to modulate immune responses have variants that occur in frequencies ranging from rare $(<1 \%)$ to common $(>10 \%)$ in the general population. There are approximately 907.3 million SNPs catalogued in the Single Nucleotide Polymorphism Database (dbSNP) build 150 (Feb 3, 2017), and it is practically impossible to systemically evaluate all polymorphic SNPs in the human genome through association studies alone. Here, we demonstrate for the first time that by examining the cancer-associated germline receptor variants that enhance the amplitude of STAT3 signaling 


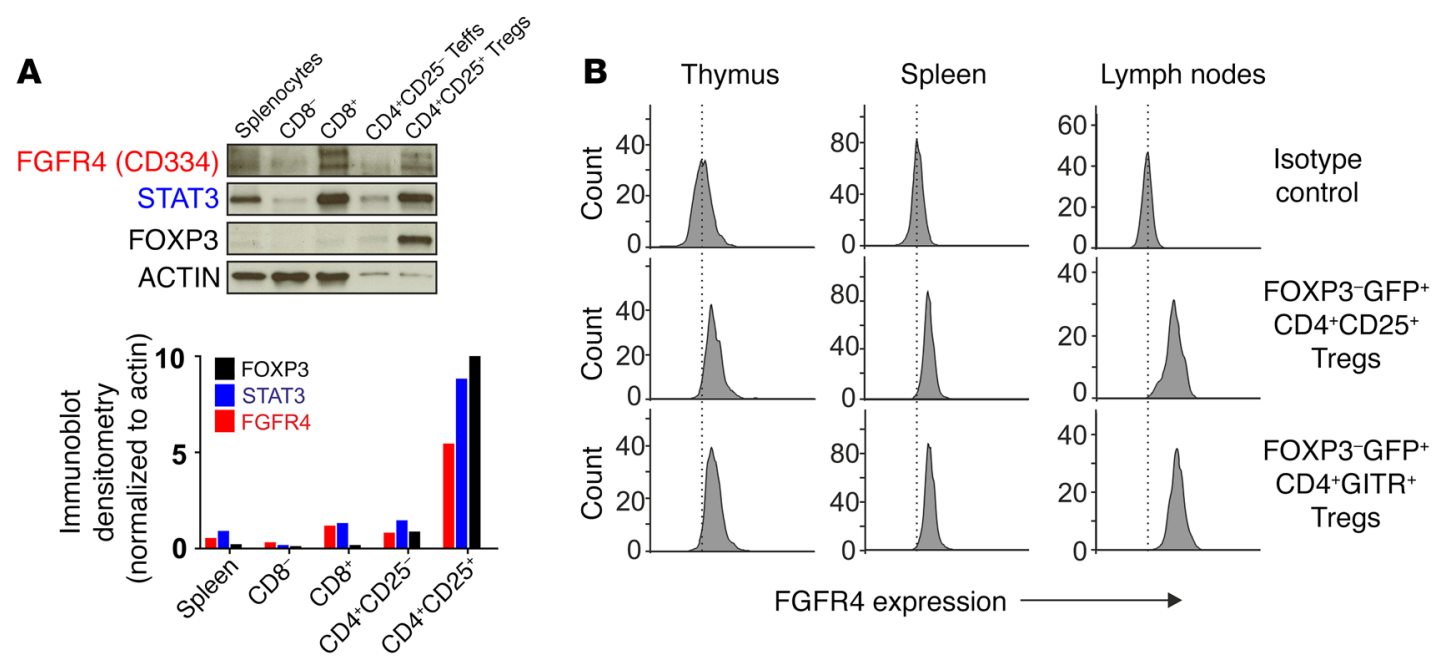

Figure 1. Expression analysis of FGFR4 in lymphocytes. (A) Representative immunoblot analysis of FGFR4, STAT3, and FOXP3 proteins in purified and pooled lymphocytes ( $n=13$ adult mice). The histogram shows digital quantification normalized to actin expression bands. The data shown are representative of 3 independent cell isolation and immunoblot experiments. (B) Expression analysis for FCFR4 in CD4+CD25+ and CD4 $4^{+} \mathrm{CITR}{ }^{+}$regulatory lymphocytes using fluorochrome-conjugated antibodies in blood, mesenteric lymph nodes, spleen, and thymus isolated from 7-month-old Foxp3-GFP reporter mice. Plots are representative of 5 independent biological replicates.

in a genotype-dependent manner (7), potential individual-specific modulators of cancer immune surveillance can be systematically evaluated. Amplified STAT3 signaling is promitotic in cancer cells, whereas studies using targeted ablation of STAT3 signaling in immune cells, such as DCs, CD8 T cells, regulatory T cells, NK cells (8), and macrophages (9-11), establish the immunosuppressive properties of constitutively activated STAT3. We hypothesized that STAT3-enhancing germline variants are potentially the tumor-extrinsic germline-encoded determinants of immune evasion in the TME. Our work provides valuable insights into the predictive value of host-specific STAT3-enhancing germline variants in impeding the immune cell infiltration of tumors.

\section{Results and Discussion}

To identify all SNP variants that create membrane-proximal tyrosine-based STAT3 docking motifs, we performed a comprehensive computational analysis of all publicly available human genotyping data sets, namely, the 1,000 Genomes, Phase 3 (12), the Catalogue of Somatic Mutations in Cancer (COSMIC) (13), the Cancer Cell Line Encyclopedia (CCLE) (14), the dbSNP (15), the Exome Aggregation Consortium (ExAC.rO.3) (16), the International HapMap Project (HAPMAP) (17), the United States National Cancer Institute (NCI) 60 human tumor cell line (NCI60) exomes (18), the Cancer Genome Atlas (TCGA) (19), and the whole genome sequences of healthy elderly people (Wellderly) (20), using our new python-based algorithm called the Transmembrane Protein Sequence Variant Identifier (TraPSVarI) (21). We reviewed the approximately one billion human variants analyzed, and identified SNPs in the human variome (Supplemental Table 1; supplemental material available online with this article; https://doi.org/10.1172/JCI96708DS1) that create membrane-proximal STAT3 binding sites in juxtamembrane segments. Interestingly, a large majority of these rare SNPs in type I membrane proteins exhibited expression patterns restrict- ed to either professional antigen-presenting cells (namely,

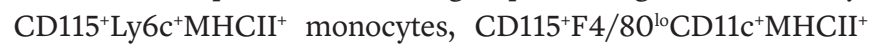
SiglecF- macrophages, or $\mathrm{CD}^{-} 5^{+} \mathrm{CD} 11 \mathrm{~b}^{+} \mathrm{CD} 11 \mathrm{c}^{+} \mathrm{MHCII}^{+} \mathrm{DCs}$ ) or immunosuppressive $\mathrm{CD}^{+} \mathrm{CD}^{+} \mathrm{CD} 25^{+} \mathrm{FOXP}^{+}$regulatory $\mathrm{T}$ cells (Supplemental Table 1 and Supplemental Figure 1, A-C; see complete unedited blots in the supplemental material). The SNP allele rs351855-A encoding the fibroblast growth factor receptor 4 variant (FGFR4 p.Gly388Arg) is the only commonly occurring STAT3-enhancing receptor variant with a minor allele frequency of 0.3 in the general population. Among the immune cells profiled, FGFR4 (alias CD334) was highly expressed in $\mathrm{CD}^{+}{ }^{+} \mathrm{CD} 25^{+} \mathrm{FOXP}^{+}{ }^{+}$Tregs (Figure 1A; see complete unedited blots in the supplemental material). Using FOXP3-GFP-knockin reporter mice (22), we found that protein levels of FGFR4 in $\mathrm{CD}^{+} \mathrm{CD}^{+} 5^{+} \mathrm{FOXP}^{+}{ }^{+}$or $\mathrm{CD}^{+}{ }^{+} \mathrm{GITR}^{+} \mathrm{FOXP}^{+}$Treg populations are elevated when Tregs are resident in the lymph nodes (Figure 1B and Supplemental Figure 2). Here, using transgenic rs351855 SNP-knockin mice (homozygous for minor allele rs351855-A denoted hereafter by $\left.F g f r 4^{r 3551855-A / A}\right)$ and their WT littermates (homozygous denoted by Fgfr $4^{r 3551855-G / G}$ ), we asked whether STAT3-enhancing germline variants can shape the TMEs pleiotropically independent of the cancer types. We first ascertained that the minor allele variant rs351855-A was functional in Tregs as indicated by elevated levels of 705-tyrosine phosphorylated STAT3 (pY705) (Supplemental Figure 3, A-D; see complete unedited blots in the supplemental material) in $\mathrm{Fgfr}^{\mathrm{r} 3351855-\mathrm{A} / \mathrm{A}}$ mice. No obvious differences were detected when we monitored Fgfr $4^{r 3351855-A / A}$ - and Fgfr $4^{r 3551855-G / G}$-knockin mice at 5 to 6 months of age by assessing the proportions of monocytes/macrophages and $\mathrm{B}$ and $\mathrm{T}$ lymphocytes in lymphoid compartments, including bone marrow, thymus, blood, lymph nodes, and spleen (Supplemental Figure 4, A-C). Likewise, analyses for the proportions of NK cells and TCR $\gamma \delta^{+} \mathrm{T}$ cells in the thymus, spleen, and lymph nodes of Fgfr $4^{r 3551855-G / G}$ and Fgfr $4^{r 3351855-A / A}$ mice (Supplemental Figure 5, 


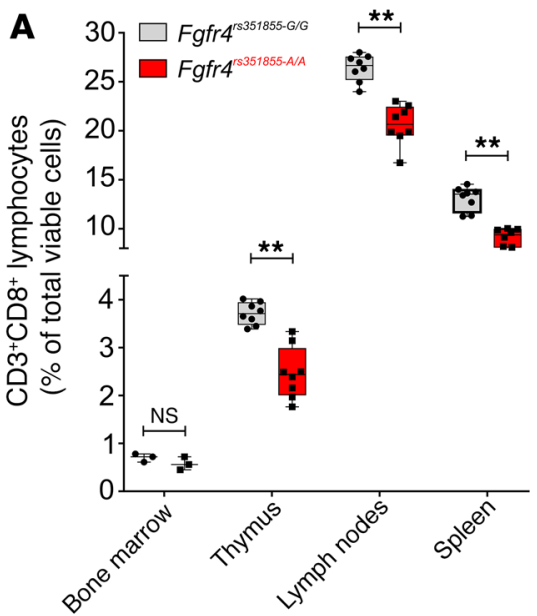

B
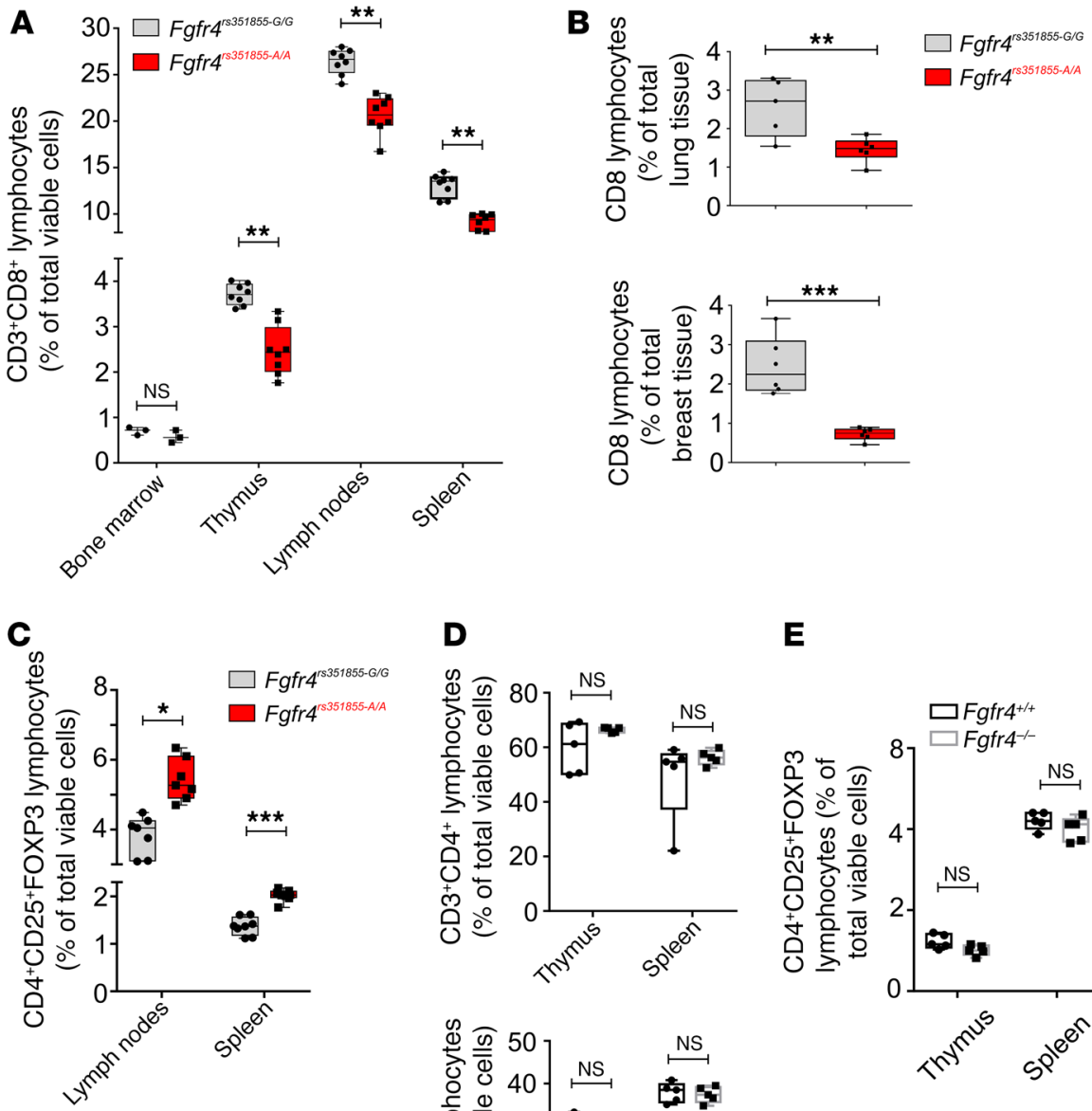

$\mathbf{E}$

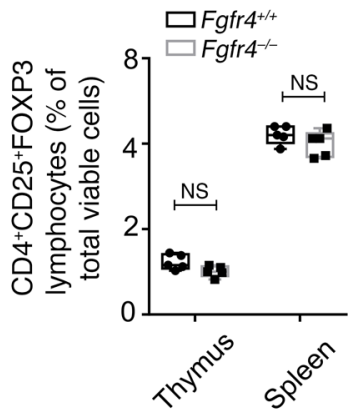

D

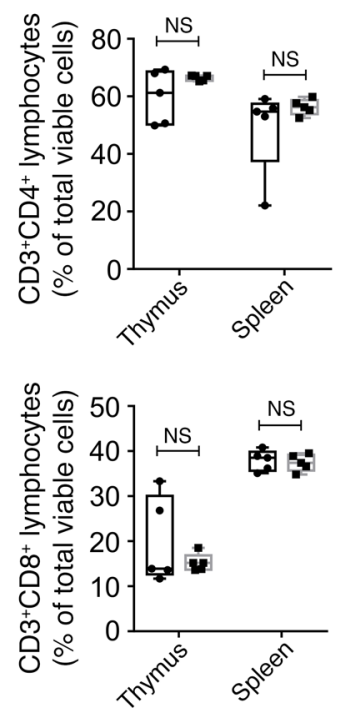

Figure 2. Rs351855 SNP-specific suppression of the CD8/Treg ratio in healthy tissues. (A) Analysis of $\mathrm{CD} 8^{+} \mathrm{T}$ cells in bone marrow, thymus, blood, lymph nodes, and spleen of 6 - to 8-week-old Fgfr4 ${ }^{\text {rs351855-A/A }}$ and Fgfr4 ${ }^{\text {r5351855-G/C }}$ mice quantified by flow cytometry. Data represent the percentages of the total single-cell suspension (mean \pm SEM, $n=5-8,{ }^{* *} P<0.01, \mathrm{NS}=$ not significant). Statistical comparisons of groups were performed using 2-way ANOVA and Tukey's $t$ test with multiple comparisons. (B) Analysis of the $\mathrm{CD}^{+} \mathrm{T}$ cell content in the nonlymphoid parenchymal organs in Fgfr4 ${ }^{\mathrm{r} 351855-\mathrm{A} / \mathrm{A}}$ and Fgfr4 ${ }^{r 3551855-c / c}$ mice. Lung samples were from 5-month-old mice (mean $\pm \mathrm{SEM}, n=6-8,{ }^{*} P<$ 0.01 ) and breast tissue was taken from mice 3 months after pregnancy (mean $\pm \mathrm{SEM}, n=3-4$, ${ }^{* *} P<0.001$ ). Infiltrating cells were measured by preparing single-cell suspensions. (C) Analysis of $\mathrm{CD}^{+}{ }^{+} \mathrm{CD} 25^{+} \mathrm{FOXP3}^{+} \mathrm{T}$ cell numbers by flow cytometry of live splenocytes from Fgfr $4^{\text {rS351855-A/A }}$ and Fgfr $^{r 5351855-C / c}$ mice. Data represent the percentages of total single-cell suspensions (mean \pm SEM, $\left.n=5-8,{ }^{*} P<0.05,{ }^{* * *} P<0.001\right)$. Statistical comparisons of groups were performed using 2-way ANOVA and Tukey's $t$ test with multiple comparisons. (D) Quantitative analysis of $\mathrm{CD} 4^{+}$ and $\mathrm{CD}^{+}$cells in the thymus and spleen of 6 - to 8-week-old $\mathrm{Fgfr}^{+/+}$and $\mathrm{Fgfr}^{-/-}$mice and (E) $\mathrm{CD}^{+} \mathrm{CD}^{+} 5^{+} \mathrm{FOXP3}^{+}$cells in the thymus and spleen of 6- to 8-week-old $\mathrm{Fgfr}^{+/+}$and $\mathrm{Fgfr}^{-/-}$mice measured by flow cytometry. Data represent the percentages of total single-cell suspensions (mean \pm SEM, $n=5-6$, NS = not significant). Statistical comparisons of groups were performed using 2-way ANOVA and Tukey's $t$ test with multiple comparisons. All flow cytometry measurements on WT and mutant cohorts of mice were performed on the same day.
$\mathrm{A}$ and $\mathrm{B})$ showed no significant differences between the 2 genotypes. However, the numbers of $\mathrm{CD} 8^{+} \mathrm{T}$ cells were significantly decreased in the thymus, blood, lymph nodes, and spleen of Fgfr $4^{r \text { rs51855-A/A }}$ mice compared with those of the WT Fgfr $4^{r 351855-G / G}$ littermates (Figure 2A and Supplemental Figure 6, A and B). The suppressed levels of $\mathrm{CD}^{+} \mathrm{T}$ cells in $\mathrm{Fg} f \mathrm{r}^{\mathrm{r}}{ }^{\mathrm{r} 351855-\mathrm{A} / \mathrm{A}}$ genotypes appeared as a systemic trait, since lower levels were also found in the nonlymphoid organs analyzed, including parenchymal tissues such as lung and mammary tissue pads (Figure 2B). On the other hand, the quantification of FOXP $3{ }^{+} \mathrm{CD} 25^{+}$Tregs revealed a significant increase in Tregs under unchallenged homeostasis conditions in healthy adult mice (Figure 2C and Supplemental Figure 6C). Concordantly, the levels of Foxp3 and Il10 transcripts were significantly elevated, whereas the levels of $C d 8$ mRNA transcripts were decreased in the spleens of $\mathrm{Fg} f \mathrm{r}^{\mathrm{r} 3551855-\mathrm{A} / \mathrm{A}}$ mice (Supplemental Figure 7, A-C), further supporting a general decrease in the CD8/Treg ratio in vivo. Interestingly, immunophenotyping analyses of WT $\left(\mathrm{Fgfr}^{+/+}\right)$and FGFR4-deficient $\left(\mathrm{Fgfr}^{-/}\right)$mice showed no significant alterations in the CD8/Treg ratio (Figure 2, D and E) or other immune cells analyzed, including NK cells, TCR $\gamma \delta^{+} \mathrm{T}$ cells, B cells, and macrophages (Supple- mental Figure 8, A-C) either in lymphoid or in parenchymal organs (data not shown).

To functionally consolidate our findings, we determined that an increase in PSTAT3 at Y705 in Tregs resulted in enhanced proliferation and suppressive functions of Tregs. Isolated Tregs from Fofr $4^{r \text { r351855-A/A }}$ mice exhibited a higher proliferation rate than the Fgfr $4^{r 3551855-G / G}$ Tregs, as shown by the eFluor670 dilution assay performed on CD3/CD28-activated cells ex vivo (Figure 3A), supporting STAT3-associated Treg proliferative function. However, we did not observe a similar increase in the proliferative potential of Fgfr $4^{r 3351855-A / A} \mathrm{CD} 8^{+} \mathrm{T}$ cells. To assess whether an increase in Tregs may play a role in the suppression of CD8 lymphocytes in $\mathrm{Fgfr} 4^{\mathrm{r} 3551855-\mathrm{A} / \mathrm{A}}$ mice, the functional capacities of $\mathrm{CD} 4^{+} \mathrm{CD} 25^{+}$ Tregs of either genotype in suppressing the expansion of $\mathrm{CD} 8^{+}$ $\mathrm{T}$ cells ex vivo were determined by in vitro-activated cocultivation assays. Three days after cocultivation, Tregs derived from the spleens of Fgfr $4^{\text {rs351855-A/A }}$ mice suppressed the expansion of CFSE-labeled $\mathrm{CD} 8^{+} \mathrm{T}$ cells to a significantly larger extent than the splenic Fgfr $4^{r 3551855-G / G}$ Tregs (Figure 3B). To some degree, the suppression was dependent on interleukin 10 (IL10), since the presence of neutralizing IL10 mAb in the cocultures, particularly 

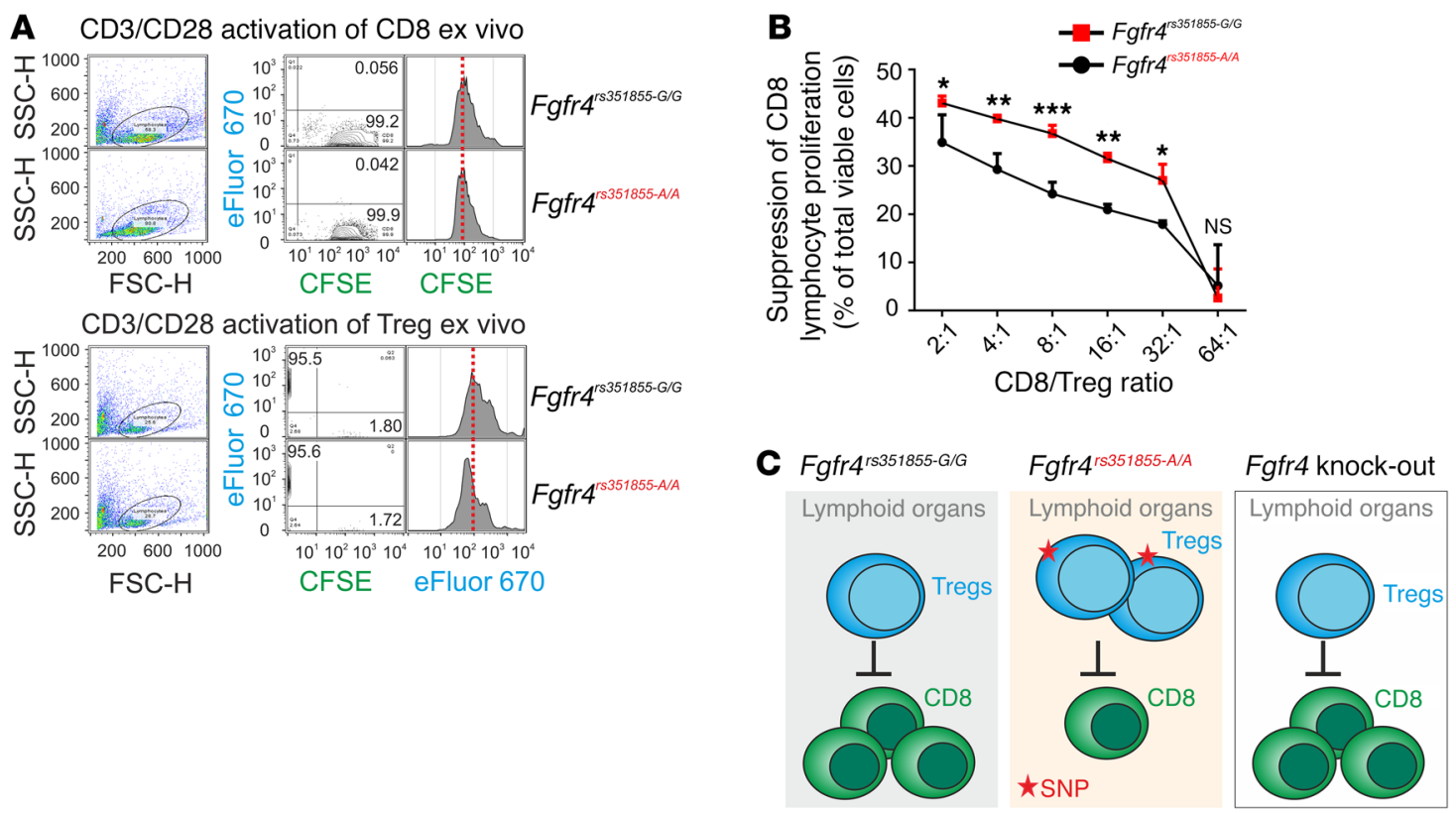

Figure 3. Genotype-specific suppression of the CD8/Treg ratio ex vivo. (A) Dye dilution assay assessing the proliferative capacities of eFluor670- and CFSE-loaded CD4+CD25+ Tregs and CD8 T cells, respectively, 72 hours after CD3/CD28 stimulation of cells isolated from spleens of $\mathrm{Fg} f \mathrm{fr}^{\mathrm{r}}{ }^{\mathrm{r} 351855-\mathrm{A} / \mathrm{A}}$ and

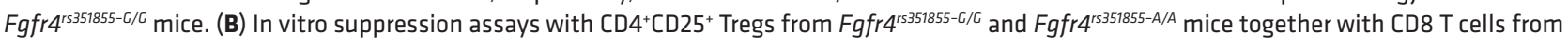
Fgfr4 $4^{r 351855-C / C}$ and Fgfr4 ${ }^{r 351855-A / A}$ mice, respectively. Tregs and CD8 T cells were mixed at different ratios and stimulated using mouse T-activator CD3/ CD28 Dynabeads. Three days after stimulation, the percentage of suppression was calculated as described in Methods. Data are representative of 3 independent experiments. For each experiment, cells were isolated from a group of 5 mice of either genotype, and each mixed ratio was cocultivated in replicates of 5 wells (mean \pm SEM, NS = not significant, ${ }^{*} P<0.05$, ${ }^{* *} P<0.01$, ${ }^{* *} P<0.001$ ). Statistical comparisons of groups were performed by 2 -way ANOVA using Sidak's multiple comparisons test. (C) Graphic summary illustrating the SNP-specific gain of immunological function by the minor allele (NC_000005.10:g.177093242G>A) of the rs351855 SNP. The genotype-dependent phenotype is abolished in the FGFR4-deficient mice and is indistinguishable from the WT FGFR4-variant-expressing cohorts, indicative of enhanced rs351855 G>A-variant-specific STAT3 signaling in lymphoid organs.

in higher CD8/Treg ratios (32:1, 16:1), led to similar suppressive capacities by both genotypes (Supplemental Figure 9). IL10 signals primarily by inducing pSTAT3 at Y705 via the STAT3 docking sites in the cytoplasmic domains of IL10R (23). Therefore, we conclude that the synergistic action of the rs351855-A allele with IL10 signaling explains the enhanced suppressive functions of Tregs in Fgfr $4^{r 3551855-A / A}-$ knockin mice. Thus, under healthy homeostatic conditions, a germline-encoded increase in basal pSTAT3 (Y705) levels in Tregs leads to a systemic decrease in the $\mathrm{CD} 8$ /Treg ratio. This finding suggests that alterations in the $\mathrm{CD} 8 /$ Treg ratio in vivo are mechanistically linked to the presence of the minor allele rs351855-A and are not determined by the activity of FGFR4. The STAT3-enhancing gain of function by the minor allele of rs351855 is independent of the extracellular or intracellular domains of FGFR4 and is mediated by the membrane-proximal STAT3 docking site in the juxtamembrane segment of the FGFR4 p.Gly388Arg variant (24). We therefore attribute the genotype-dependent systemic suppression of the $\mathrm{CD} 8 /$ Treg ratio to the pleiotropic effect of STAT3-enhancing gain of function by the SNP rs351855-A. Disruption of the STAT3 membrane-recruitment event by the depletion of FGFR4 in the Fgfr $4^{--}$mice abolished the SNP-specific gain of the immunological phenotype (Figure 3C).

To determine whether STAT3-enhancing germline variants mediate a tumor-extrinsic immune evasive pleiotropic phenotype, we generated genetically engineered SNP-knockin mouse models (GEKIMM) for breast and lung cancers (see Methods). As expected, although the tumor incidence rates in both the disease models were not dramatically altered, the tumor burden and progression were significantly elevated in animals expressing the minor allele variant rs351855-A (25). Flow cytometric analysis of the age- and sexual phenotype-matched cohorts of knockin transgenic mouse models for breast (26) ( $F g f r 4^{r 3351855-G / G} W A P-T g f a$ and Fgfr $4^{r 3551855-A / A}$ WAP-Tgfa) and lung (27) (Fgfr $4^{r 3551855-G / G}$ SPCCrafB B B and Fgfr $4^{r 3351855-A / A}$ SPC-CrafB B B) cancers revealed a significant increase in the proportions of $\mathrm{CD} 4^{+} \mathrm{CD} 25^{+} \mathrm{FOXP} 3^{+}$Tregs

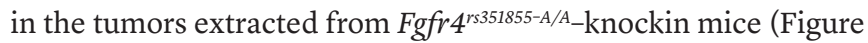
4, A and B). Furthermore, the significant increase in tumor-infiltrating Tregs correlated with increased Tregs in lymphoid organs (data not shown) and elevated serum levels of IL10 in Fgfr $4^{r 3351855-A / A}$ WAP-Tgfa (Figure 4C) and Fgfr4 $4^{r 3351855-A / A} S P C$-CrafBxB (Figure 4D) mice. On the other hand, a marked reduction in the numbers of tumor-infiltrating $\mathrm{CD}^{+} \mathrm{T}$ cells was observed in the $\mathrm{Fgfr} 4^{\mathrm{r} 3551855-\mathrm{A} / \mathrm{A}}$ cohorts of GEKIMMs for both breast (Figure 4E) and lung (Figure $4 \mathrm{~F}$ ) cancers. Although STAT3 signaling is considered crucial for $\mathrm{T}$ helper cell differentiation during immune challenges, we did not observe any significant differences between the 2 genotypes in the transgenic disease models for breast or lung cancer. The proportions of differentiated $\mathrm{CD} 4^{+} \mathrm{T}$ cell subsets (namely, Th1, Th2, and Th17) in spleens and tumors of GEKIMM for lung cancer (Supplemental Figure 10) and GEKIMM for breast cancer (Supplemental Figure 11) were not altered. We propose that the 
A

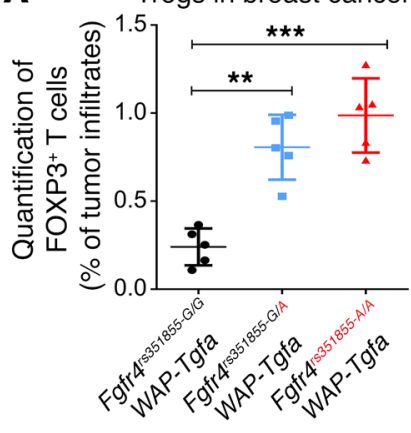

C

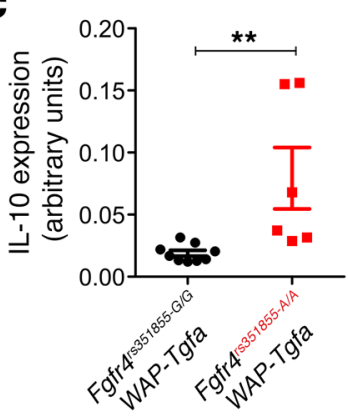

E

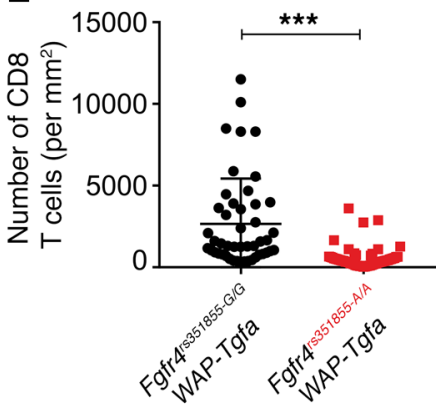

$\mathbf{F}$

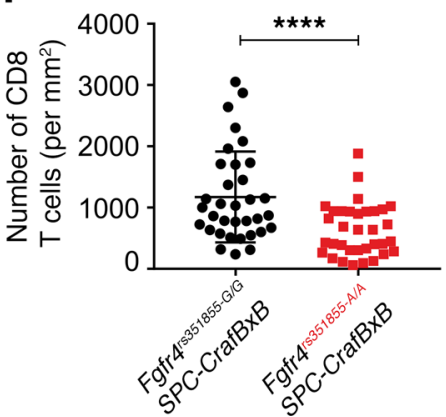

B
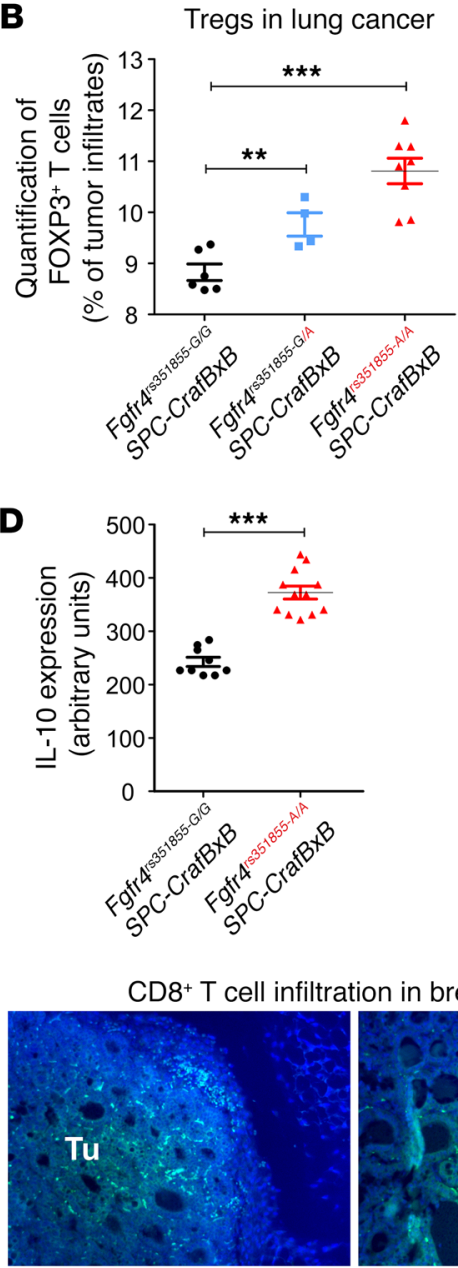

Fgfr4:5351855-6/6 WAP-Tgfa

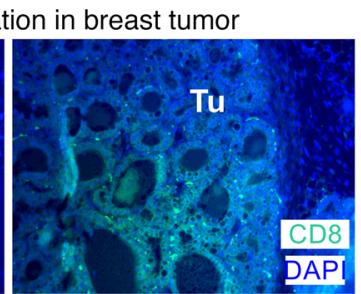

Fgfr4's351855-A/A WAP-Tgfa

Figure 4. Rs351855 SNP-specific suppression of the CD8/Treg ratio in the TME. (A) Numbers of $\mathrm{CD}^{+}{ }^{+} \mathrm{CD} 25^{+} \mathrm{FOXP3}^{+} \mathrm{T}$ cells in tumor-bearing breast tissue of Fgfr4'r355855-c/c Wap-Tgfa,

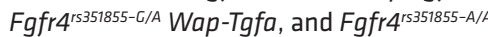
Wap-Tgfa mice (mean $\pm \mathrm{SEM}, n=5,{ }^{*} P<0.01$, $\left.{ }^{* *} P<0.001\right)$. (B) Numbers of $\mathrm{CD} 4{ }^{+} \mathrm{CD} 25^{+} \mathrm{FOXP3}^{+}$ T cells in tumor-bearing lungs of $F g f r 4^{r 5351855-C / C}$ SPC-CrafBXB, Fgfr4 ${ }^{r 5351855-C / A} S P C-C r a f B \times B$, and Fgfr4 $4^{r 3551855-A / A}$ SPC-CrafB $\times B$ mice (mean \pm SEM, $\left.n=4-7,{ }^{* *} P<0.01,{ }^{* *} P<0.001\right)$. (C) Quantification of IL10 in serum of tumor-bearing Fgfr4 ${ }^{r 5351855-G / C}$ Wap-Tgfa and Fgfr4 ${ }^{r 5351855-A / A}$ WapTgfa breast cancer mice (mean \pm SEM, $n=6-9$, $\left.{ }^{*} P<0.01\right)$ and $(\mathbf{D})$ tumor-bearing Fgfr4 ${ }^{r 5351855-C / C}$ SPC-CrafBXX and Fgfr4 ${ }^{r 5351855-A / A}$ SPC-CrafBXXB lung cancer mice (mean $\pm \mathrm{SEM}, n=8-12$, ${ }^{* *} P$ $<0.001$ ) by ELISA. ( $E$ and $\mathbf{F}$ ) Quantification of tumor-infiltrating $C D 8^{+} T$ cells in tumor nodules by immune staining for CD8 in breast tumor-

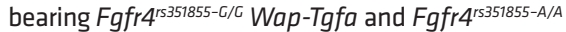
Wap-Tgfa mice and (F) lung tumor-bearing

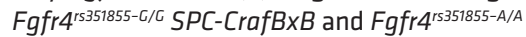
SPC-CrafBXB (mean \pm SEM, $n=19-26$, ${ }^{* * *} P<$ $0.0001,{ }^{* *} P<0.001$, 2-tailed unpaired $t$ test with Welch's correction). Insets: Representative images from immunofluorescence staining of tumor sections ( $\times 20$ magnification) depicting tumor-infiltrating $\mathrm{CD} 8^{+} \mathrm{T}$ cells in lung and breast tumors (DAPI-blue, CD8-green). The red text in the figure denotes the minor allele of the SNP rs351855.

differences in these subsets may be notable in the mouse models for inflammation-induced cancers. Collectively, through data from knockin mice, knockout mice, and genetically engineered knockin mouse models for lung and breast cancers, our study illustrates a pleiotropic effect of cancer-associated STAT3enhancing germline variants in shaping some aspects of the TME (Supplemental Figure 12). Hence, we conclude that the immune evasive phenotype of the TME can be determined by the pleiotropic functions of individual-specific germline variants in the immune cells. In this regard, cancer-associated germline recep- tor variants that enhance the amplitude of STAT3 signaling are potent modulators of tumor-intrinsic proliferative and tumorextrinsic immune evasive functions. Overall, our work provides valuable insight into the prognostic value of STAT3-enhancing germline receptor variants in the immune-excluded and immunologically ignorant tumor phenotype. Given that the germlineencoded STAT3-enhancing SNPs are particularly prevalent in the coding regions of immune cell surface markers, further work is warranted to explore their significance as predictive biomarkers for immunotherapy responses. 


\section{Methods}

A complete description of the methods and statistical analysis is provided in the Supplemental Materials.

Study approval. All of the experiments were performed under protocols approved and reviewed by the Institutional Animal Care and Use Committee at the Max Planck Institute of Biochemistry. Animal protocols and experimental procedures involving FGFR4-KO mice were approved by the Institutional Animal Care and Use Committee at the University of Miami Miller School of Medicine.

\section{Author contributions}

VKU conceived and designed the study. DK wrote the codes for the TraPS-VarI algorithm. VKU and DK performed the computation analyses. AG, CY, and CF performed the immune phenotyping analyses for the Fgfr4-knockout mice. VKU performed the experiments, analyzed the data, interpreted the results, and wrote the manuscript. The final version of the manuscript was reviewed by all the coauthors.

\section{Acknowledgments}

The authors thank Axel Ullrich (Max Planck Institute of Biochemistry) for reading the drafts of this manuscript and generously supporting this work, Kerstin Berer (Max Planck Institute of Neurobiology) and Gurumoorthy Krishnamoorthy (Max Planck Institute of Biochemistry) for advice and useful comments, and Susanne Wuchenauer and Bianca Sperl (Max Planck Institute of Biochemistry) for technical assistance. The authors acknowledge Heinz Brandstetter and Corrina Moerth (Max Planck Institute of Biochemistry) for their services with the animal housing facilities. AG and $\mathrm{CF}$ are supported in part by the American Heart Association. CF is supported by the American Diabetes Association (1-16-IBS087) and by the NIH (R01HL128174).

Address correspondence to: Vijay K. Ulaganathan, Max Planck Institute for Biochemistry, Department of Molecular Biology, Am Klopferspitz 18, 82152, Martinsried, Germany. Phone: 49.89.8578.2511; Email: ulaganat@biochem.mpg.de.
1. Hanahan D, Weinberg RA. Hallmarks of cancer: the next generation. Cell. 2011;144(5):646-674.

2. Hoos A. Development of immuno-oncology drugs - from CTLA4 to PD1 to the next generations. Nat Rev Drug Discov. 2016;15(4):235-247.

3. Palucka AK, Coussens LM. The basis of oncoimmunology. Cell. 2016;164(6):1233-1247.

4. Fridman WH, Pagès F, Sautès-Fridman C, Galon J. The immune contexture in human tumours: impact on clinical outcome. Nat Rev Cancer. 2012;12(4):298-306.

5. Kroemer G, Galluzzi L, Kepp O, Zitvogel L. Immunogenic cell death in cancer therapy. Annu Rev Immunol. 2013;31:51-72.

6. Dunn GP, Old LJ, Schreiber RD. The three Es of cancer immunoediting. Annu Rev Immunol. 2004;22:329-360.

7. Ulaganathan VK, Ullrich A. Membraneproximal binding of STAT3 revealed by cancerassociated receptor variants. Mol Cell Oncol. 2016;3(3):e1145176.

8. Wang $\mathrm{T}$, et al. Regulation of the innate and adaptive immune responses by Stat-3 signaling in tumor cells. Nat Med. 2004;10(1):48-54.

9. Cheng F, et al. A critical role for Stat 3 signaling in immune tolerance. Immunity. 2003;19(3):425-436.

10. Sun Z, Yao Z, Liu S, Tang H, Yan X. An oligonucleotide decoy for Stat 3 activates the immune response of macrophages to breast cancer. Immunobiology. 2006;211(3):199-209.

11. Yu H, Kortylewski M, Pardoll D. Crosstalk between cancer and immune cells: role of STAT3 in the tumour microenvironment. Nat Rev Immunol. 2007;7(1):41-51.

12. 1000 Genomes Project Consortium, et al. A global reference for human genetic variation. Nature. 2015;526(7571):68-74.

13. Forbes SA, et al. COSMIC: exploring the world's knowledge of somatic mutations in human cancer. Nucleic Acids Res. 2015;43(Database issue):D805-D811.

14. Barretina J, et al. The Cancer Cell Line Encyclopedia enables predictive modelling of anticancer drug sensitivity. Nature. 2012;483(7391):603-607.

15. Sherry ST, et al. dbSNP: the NCBI database of genetic variation. Nucleic Acids Res. 2001;29(1):308-311.

16. Lek M, et al. Analysis of protein-coding genetic variation in 60,706 humans. Nature. 2016;536(7616):285-291.

17. International HapMap Consortium. The International HapMap Project. Nature. 2003;426(6968):789-796.

18. Reinhold WC, et al. NCI-60 whole exome sequencing and pharmacological CellMiner analyses. PLoS One. 2014;9(7):e101670.

19. The Cancer Genome Atlas Research Network, et al. The Cancer Genome Atlas Pan-Cancer analysis project. Nat Genet. 2013;45(10):1113-1120.

20. Erikson GA, et al. Whole-genome sequencing of a healthy aging cohort. Cell. 2016;165(4):1002-1011

21. Kogan D, Ulaganathan VK. TraPS-VarI: a python module for the identification of STAT3 modulat- ing germline receptor variants. bioRxiv. https:// doi.org/10.1101/173047.

22. Kuczma M, et al. Foxp3-deficient regulatory $\mathrm{T}$ cells do not revert into conventional effector CD4 ${ }^{+} \mathrm{T}$ cells but constitute a unique cell subset. Jimmunol. 2009;183(6):3731-3741.

23. Weber-Nordt RM, Riley JK, Greenlund AC, Moore KW, Darnell JE, Schreiber RD. Stat3 recruitment by two distinct ligand-induced, tyrosine-phosphorylated docking sites in the interleukin-10 receptor intracellular domain. J Biol Chem . 1996;271(44):27954-27961.

24. Ulaganathan VK, Sperl B, Rapp UR, Ullrich A. Germline variant FGFR4 p.G388R exposes a membrane-proximal STAT3 binding site. Nature. 2015;528(7583):570-574.

25. Seitzer N, Mayr T, Streit S, Ullrich A. A single nucleotide change in the mouse genome accelerates breast cancer progression. Cancer Res. 2010;70(2):802-812.

26. Sandgren EP, Schroeder JA, Qui TH, Palmiter $\mathrm{RD}$, Brinster RL, Lee DC. Inhibition of mammary gland involution is associated with transforming growth factor alpha but not c-myc-induced tumorigenesis in transgenic mice. Cancer Res. 1995;55(17):3915-3927.

27. Kerkhoff E, Fedorov LM, Siefken R, Walter AO, Papadopoulos T, Rapp UR. Lung-targeted expression of the c-Raf- 1 kinase in transgenic mice exposes a novel oncogenic character of the wild-type protein. Cell Growth Differ. 2000;11(4):185-190. 